\title{
An Approach to Create Multiple Versions of the Same Learning Object
}

\author{
http://dx.doi.org/10.3991/ijet.v9i5.3762 \\ Y.Z. Seghroucheni ${ }^{1}$, A. Mohammed ${ }^{2}$ and B.E. El Mohajir ${ }^{1}$ \\ ${ }^{1}$ Faculty of Sciences, Tetouan, Morocco \\ ${ }^{2}$ The National Applied School of Sciences, Tetouan, Morocco
}

\begin{abstract}
-several studies have shown that learning is effective when it takes into consideration the characteristics and learning styles of learners. The existing systems generate learning paths based on the learning styles, and by studying the behaviors within the same system, to create the accurate learning path, while playing mostly with the order of learning objects presented (according to prerequisites, objectives,...). However these systems do not act neither on the cognitive level of the knowledge itself nor its didactic aspects. It is assumed that the proposed learning objects are valid for all learners, and it's only a matter of suitable sequence for everyone. Our aim is to propose a model of contents, based on the differentiated pedagogy, to produce multiples versions of the same course, taking into account the different requirements of differentiated pedagogy. We propose an approach that consists of differentiating the available educational resources, by creating multiple versions of the same learning object to ensure its better assimilation.
\end{abstract}

Index Terms-learning styles, learning path, learning objects, differentiated pedagogy.

\section{INTRODUCTION}

The use of Internet for educational purposes is currently growing in many forms, especially in universities. This teaching method allowing the learner to be the actor of his training, is basically offering training according to his own pace regardless of other learners, and more adapted to his own learning profile. Most intelligent system that exist in eLearning [1]-[11] are interested into adapting learning to the different profiles. The logic to this is that the accommodation of differences in content to learners, prerequisites, objectives and preferences [16] improves the system performances, which translates subsequently to a more satisfactory result on the learner's side. However those systems provide the same learning object for everyone, because they do not take into account the differences that may appear at the cognitive level or the didactic aspect of the learning object.

In this paper, we present an approach that consists of creating multiple versions of the same learning object based on the differentiated pedagogy; it aims to offer a variation of educational resources of the same LO to ensure its better assimilation by all the learners.

The rest of this paper is organized as follows: We start in section 2 with a brief review of related works, and then in section 3 we will present the multiple definitions of the learning objet. In section 4 we will present the differentiated pedagogy, and then we will discuss the adaptation of the course and its structure in section 5. Later we will propose our approach for creating different versions of the same LO based on the differentiated pedagogy in section 6. Finally some conclusions are drawn in section 7.

\section{RELATED WORK}

Learning objects are the main focus of a vast majority of works in the field of adaptive learning systems; as [23] argues that a new generation of powerful e-learning system would start on the crossroads of two emerging fields: courseware re-use and adaptive educational systems, [29][20] also examine the learning object granularity [21] issue which is directly related with Learning Object (LO) reusability and the adaptability process required in Adaptive learning system.

The Reusability [8], addressed in [25] where the authors agree that LOs are the answer to sharing, re-using and locating educational materials, is the main focus also in [19], where the authors propose a system called SLDF designed to support the development of pedagogically sound learning material within an integrated platform independent data structure. The system supports sharing, re-use and adaptation of learning material. In [31] the authors classify some basic assessment aspects (adaptivity parameters) of an AEHS user, extracted from research work that refers to commonly accepted e-learning standards, such as SCORM. Also, authors provide a starting point for the development of a generic architecture for the retrieval of standardized Learning Objects (LOs) from disperse Learning Objects Repositories (LORs) to an elearning environment.

[32] Presents a new structure for Multi-Adaptive Learning Object Repository (MALOR) that is oriented towards unified Web-based educational systems. In [11] the author points out the difficulties and the opportunities of learning objects [4] where metadata is a key element [6].

The authors of [21] developed an object identification system called Active Atlas, which compares the objects shared attributes in order to identify matching object. Finally [30] points out the problem that LOM are an XML-Based Development, which emphasizes on syntax and format and is not semantically driven by knowledge representation.

The presentation of the works above shows clearly that efforts are jointed towards learning objects within the adaptive systems. Most of the systems focus on two aspects of LO: reusability and granularity. On the other hand those adaptive systems offer learning objects in an order suitable for learners which create a sort of learning path that respect their objectives, perquisites... None of those works presented above questioned the cognitive level of 
the learning object, it is assumed that the proposed learning objects are valid for all learners, and it's only a matter of suitable sequence for everyone.

\section{LEARNING OBJECT}

Most proposed definitions of learning object focus on the general principles governing concept of LO such as: reusability in different situation for learning and the independence of context [28]. Balatsoukas [17] gives a typical example of the Polsani definition [8]. This author defines a LO as a unit of content Learning independent and autonomous, which is predisposed to be reuse in multiple learning contexts. Other authors such as Bibeau [18] considers LO as the smallest information unit or the smallest processing tool information (or applications software) used in an educational context with an intention teaching for learning through the media technology. Flamand [14] identifies three categories of LO. He distinguishes objects with little media complex and context-free (video speech of a head of state radio interview, etc.) utilitarian (modeling software, etc.) and LOs consisting of elements basic information (facts, ideas, concepts, principles, processes).

Finally, other approaches such as those of Downes [9] consider the LO size as important. Barron [7] trying to consider this approach, suggests that five to nine information objects (text, image, video, photos, etc.) can be combined to form a LO. Other works of Mortimer [24] undertaken in this direction approach the LO size in terms of time. A LO takes no longer than 15 minutes to complete.

In addition to these theoretical conceptualizations and sometimes ambiguous, other definitions emerge from various works on standardization (SCORM, LOM, IMS, etc.). For IEEE Learning Technology Standards Committee, LO are defined as any entity, digital or non-digital, which can be used, re-used or referenced during technology supported learning. Normetic [10] adds to this definition the technological support that covers the multimedia content, content instruction, educational software and software tools mentioned in a learning context to support technology. Finally, the center of Wisconsin online resources [26] defines a LO as "small learning units with a duration between 2 and 15 minutes".

Similar efforts to develop a common conceptual definition of LOs have yet to emerge. There is a broad understanding among the members of the LO community about the functional requirements of LOs:

- Accessibility: the LO should be tagged with metadata so that it can be stored and referenced in a database.

- Reusability: once created, a LO should function in different instructional contexts.

- Interoperability: the LO should be independent of both the delivery media and knowledge management systems.

\section{A. The multiple definitions of a learning object}

Currently, there are as many definitions of LOs as there are users. Here is a small sample:

1. "For this standard (Draft Standard for Learning Object Metadata v6.1), a Learning Object is defined as any entity, digital or non-digital, that may be used for learning, education or training" (IEEE Learning Technology Standards Committee 2001)
2. "...a Learning Object... [is] 'any digital resource that can be reused to support learning.' This definition includes anything that can be delivered across the network on demand, be it large or small. Examples of smaller reusable digital resources include digital images or photos, live data feeds (like stock tickers), live or prerecorded video or audio snippets, small bits of text, animations, and smaller web-delivered applications, like a Java calculator. Examples of larger reusable digital resources include entire web pages that combine text, images and other media or applications to deliver complete experiences, such as a complete instructional event" [11].

3. "Learning Objects are a new way of thinking about learning content. Traditionally, content comes in a several hour chunk. Learning Objects are much smaller units of learning, typically ranging from 2 minutes to 15 minutes [25].

4. "[A Learning Object] is defined as the smallest independent structural experience that contains an objective, a learning activity and an assessment." [2].

\section{B. Metada for learning object}

Metadata is usually defined as "data about data", any kind of information that in some way references or describes aspects of some other piece of information. Metadata is introduced in information management systems in order to support certain administrative operations, including searching, displaying summaries or configuring interfaces. In essence, metadata creates a level of indirection, allowing systems to manage resources without even having to delve into their physical or digital internals.

In an e-learning context, metadata may consist of many kinds of information about a learning object, from descriptions and subject classifications to accessibility characteristics and relations between learning objects. For example learning objects metadata may be used by cataloguing software for indexing, by learning management systems for matching learners with relevant resources, and by content players that configure the learning object to the user's environment and needs.

A robust metadata set would contain informations pertaining to areas such as object lifecycle, technical requirements, educational specifications, copyright and classification. When looking for learning objects in a repository, it is the information contained in the metadata that is searched. Therefore consistency in specification and application of metadata, across an organization or community, facilitates searching. Having said that, a learning object can be summarized as follows:

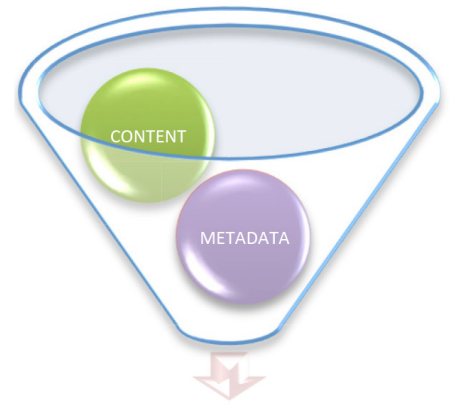

LEARNING OBJECT

Figure 1. A learning Object 
A learning object is combination of the content and the metadata, here is below the key elements of a metadata.

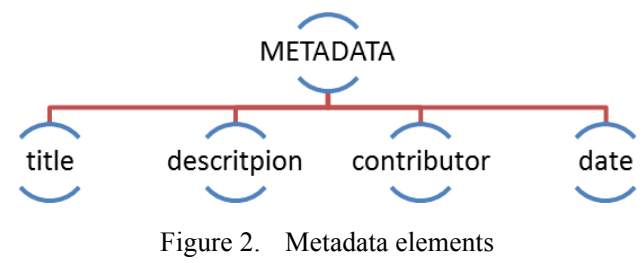

\section{DIFFERENTIATED PEDAGOGY}

To differentiate is to break with a pedagogy that is frontal, the same lessons, the same exercises etc...for all learners. The goal is to put everyone in an optimal learning situation. This organization is to use all the educational resources available so that each learner is constantly or at least very often confronted with the most fruitful teaching situations for him.

\section{A. Aspects of differentiation}

According to Philippe Meiriu [3], it is essential to define a space in which the learning activity can be exercised. A learning situation is built around three intertwined poles that are the learner, teacher and knowledge. Meirieu emphasizes that the failure of some learning situations often is that it attaches importance to the two components which are knowledge and teaching at the expense of the third which is nevertheless the platform of the whole building. The practice of differentiated pedagogy must consider each of these three areas, and its success depends heavily on how they interacted.

\section{B. Differentiation of content}

The content of lessons may be differentiated based on what learners already know. The most basic content of a lesson should cover the standards of learning set by the district or state. Some learners may be completely unfamiliar with the concepts in a lesson, while others may have partial mastery of the content - or display mistaken ideas about the content, and some others may show mastery of the content before the lesson begins. The differentiation of the content could happen by designing activities for groups of learners that cover different areas of Bloom's Taxonomy. For example, those who are unfamiliar with the concepts may be required to complete tasks on the lower levels of Bloom's Taxonomy: knowledge, comprehension, and application. Learners with partial mastery may be asked to complete tasks in the application, analysis and evaluation areas, and students who have high levels of mastery may be asked to complete tasks in evaluation and synthesis.

How to differentiate content?

a. Offer a variety of texts.

b. Use a variety of multimedia resources.

c. Extend the level of the didactic transposition;

d. Give more details of knowledge.

e. Etc....

\section{The Adaptation of The Course}

In this section, we will discuss how a course should be structured; the essential elements of a course are detailed in the diagram below (Figure 3).

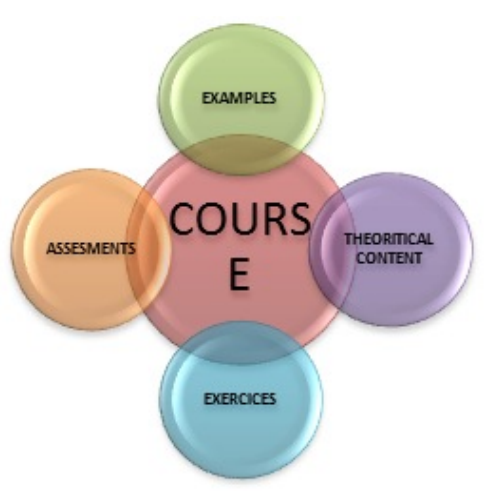

Figure 3. Key elements of a course.

A course is a combination of 4 elements, examples, assessments, exercises and the theoretical content.

\section{A. The structure of the course}

The course will contain multiple versions for each learning object, it is therefore a quadruplet:

$$
\text { COURSE }=\{\text { EXRC, EXMP, THCON, ASMT }\}
$$

\section{Where:}

EXRC represents a set of exercises (ER), each exercise is presented in different versions.

EXMP is a set of examples (EM), each example is presented in different versions.

THCON andASMT represent respectively a set of theoretical content (TC) and assessments (AS), where each one is presented in different versions.

$$
\begin{aligned}
\mathrm{EXRC} & =\sum_{\mathrm{i}}^{\mathrm{n}} \mathrm{ER} \text { where } \mathrm{ER}=\bigcup_{i}^{n} V i \\
\mathrm{EXMP} & =\sum_{\mathrm{i}}^{\mathrm{n}} \mathrm{EM} \text { where } \mathrm{EM}=\bigcup_{i}^{n} V j \\
\mathrm{THCON} & =\sum_{i}^{\mathrm{n}} \mathrm{TC} \text { where } \mathrm{TC}=\bigcup_{i}^{n} V k \\
\mathrm{ASMT} & =\sum_{\mathrm{i}}^{\mathrm{n}} \mathrm{AS} \text { where } \mathrm{AS}=\bigcup_{i}^{n} V l
\end{aligned}
$$

Where $\mathrm{V}_{\mathrm{i}}, \mathrm{V}_{\mathrm{j}}, \mathrm{V}_{\mathrm{k}}$ and $\mathrm{V}_{1}$ are the different versions available for each learning object.

The main reason behind the multiple versions of the same course is the differentiated pedagogy, which is according to [12] an approach of implementing a diverse set of resources and teaching procedures and learning, to enable students of different ages, abilities, skills and heterogeneous know-how to achieve, by different routes, common objectives and ultimately academic success.

Also, to differentiate for [14] is to implement a flexible framework where learning is explicit enough and diversified so that students can work on their own routes of ownership, while remaining in a collective educational process of required knowledge and expertise, which also aligns exactly with what suggests [13].

Finally the mathematical representation of the course is:

$$
\text { COURSE }=\sum_{i}^{n}\left(\bigcup_{i}^{n} V i+\bigcup_{j}^{n} V j+\bigcup_{k}^{n} V k+\bigcup_{l}^{n} V l\right)
$$




\section{B. The graphical representation of the course}

We give in the chart below, a graphical representation of a course.

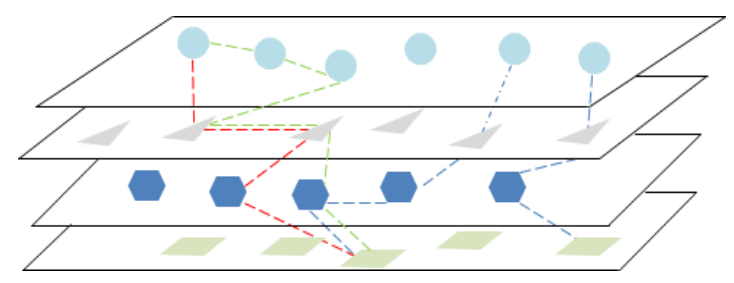

Figure 4. Example of a course.

The shapes in every level refer to the different versions of a learning object, the layers represent ether the exercises, examples, assessments or the Theoretical content. The dashed lines represent some potential learning paths.

\section{The Multiple Versions of The SAME LO}

Taking into consideration the recommendation of the differentiated pedagogy, and the course model proposed in section 5 , we choose to emphasize the following versions of a learning object:

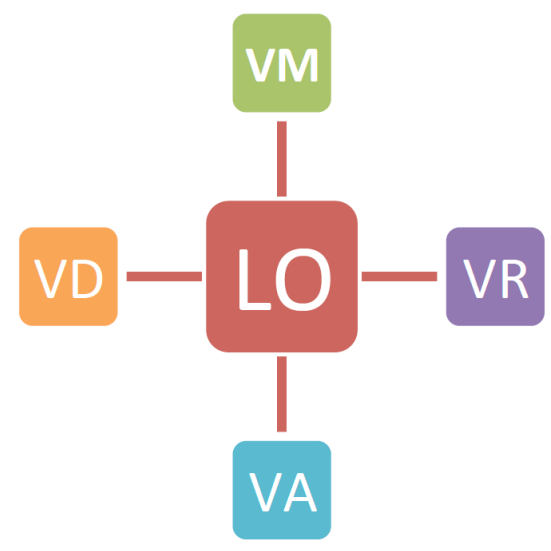

Figure 5. Versions of the same LO.

As the chart above shows, there are 4 different versions for the same LO: VM, VR, VD and VA

a. VM: a multimedia version.

b. VR: a version with a reminder of the previous LO.

c. VD: a version with a deeper level of knowledge.

d. VA: a standard version.

Having presented a learning object as above, new informations on the metadata should be added. The presentation of the metadata presented in the section 3 becomes:

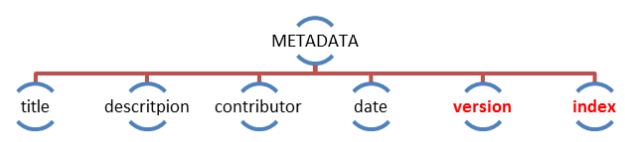

Figure 6. Metadata elements after adding new versions.

As presented in the previous diagram, there are new elements added to the metadata; version: this attribute contains the LO version (VM, VD..), while index will increment according to the different hits for the learning object, this index will help to not overload the system, as it will serve for the future to eliminate from the system the versions with a low value of entry.

\section{CONCLUSION}

We presented in this paper an approach for creating multiple versions of the same learning object based on the differentiated pedagogy, we emphasized four types of versions that are, VM, VD, VA, VR, those versions are extensible in the system, we also introduced new informations in the metadata: version and index that are useful to measure which versions have the most hits, in order to remove less popular versions to avoid overloading the system. This approach will be tested using the Chamilo platform, which is an open source e-learning platform, and the course used for this experiment is the algorithmic / C language.

\section{REFERENCES}

[1] Peter Brusilovsky, Methods and techniques of adaptive hypermedia, User Modeling and User Adapted Interaction, 1996, v 6, n 23, pp 87-129. http://dx.doi.org/10.1007/BF00143964

[2] L'Allier, James J. Frame of Reference: NETg's Map to the Products, Their Structure and Core Beliefs. NetG, 1997.

[3] Philippe Meiriu, Apprendre... oui, mais comment?, ESF éditeur, Paris, $1987,17^{\circ}$ edition, 1999.

[4] Albert Ip and al. "learning objects in different pedagogical paradigm", 2001.

[5] Sheila Tejada and al." Learning object identification rules for information integration", Information Systems Vol. 26, No. 8, 2001, pp. 607,633 .

[6] Mikael Nilsson and al. "The future of learning object metadata interoperability", ECI Conference on e-Technologies in EngineerinG, 2002.

[7] T. Barron, "Learning object approach is making inroads", In Learning Circuits, 2002.

[8] polsani, "use and abuse of reusable learning objects", Texas Digital Library, Vol.3, NO.4, 2003.

[9] S. Downes, "Design and reusability of learning objects in an academic context: A new economy of education", JSDLA Journal, 2003.

[10] Normetic, "La description normalisée des ressources : vers un patrimoine éducatif", CREPUQ Novasys Inc., 2003.

[11] David wiley,"Learning Objects: Difficulties and Opportunities", 03 april 2003.

[12] Conseil Suprieur de l'éducation, (CSE, 2001 dans Caron, 2003.

[13] Les inégalités et la différenciation de l'enseignement, Faculté de psychologie les et des sciences de l'éducation, Université de Genève, 2003.

[14] P. Flamand, A. Gervais, "Les objets d'apprentissage : au-delà de la technologie, la pédagogie", Le bulletin Clic, 2004, N54, pp 1-5.

[15] Sadat, H., \& Ghorbani, A. A. (2004), On the Evaluation of Adaptive Web Systems. Proceedings of the Workshop on Web-based Support Systems, 2004, 127-136.

[16] Halina Przesmycki, la pdagogie diffrencie, 2004, Hachette.

[17] I.F. Silveira, "Reusability and Interoperability of Adaptive Learning Objects Repositories" Symposium de l'informatique en education, 2005.

[18] R. Bibeau, "Les TIC à l'école : proposition de taxonomie et analyse des obstacles à leur intégration", EpiNet $n^{\circ} 79$, novembre 2005.

[19] J. Lukasiak, learning object and learning designs: an integrated system for reusable, adaptive and shareable learning content, 2005.

[20] Álvaro Reis Figueira and Michael Kaufmann, "disaggregation and reutilization of sharable content object", 2006 .

[21] Jeeheon Ryu and al, "Designing adaptive learning objects", 2006.

[22] Brusilovsky, P., Milln, E. User models for adaptive hypermedia and adaptive educational systems. In: Brusilovsky, P., Kobsa, A., Neidl, W. (eds.): The Adaptive Web: Methods and Strategies of Web Personalization. Lecture Notes in Computer Science, Vol. 4321. Springer- Verlag, Berlin Heidelberg New York, 2007. http://dx.doi.org/10.1007/978-3-540-72079-9 1 
PAPER

AN APPROACH TO CREATE MULTIPLES VERSIONS OF THE SAME LEARNING OBJECT

[23] Peter Brusilovsky, "From Learning Objects to Adaptive Content Services for ELearning, 2008.

[24] L. Mortimer, "Learning objects of desire: Promise and practicality". Learning Circuits, 2008.

[25] Miguel Latorre and al, "An experience on learning object reutilization based on educational resources developed, 2009.

[26] WISC, (2009) "Visconsin on line Ressource center"

[27] Battou Amal, "The granularity approach of learning objects to support adaptability in adaptive learning systems, Journal of Theoretical and Applied Information Technology, 2010.

[28] A. Battou, A. El Mezouary, C. Cherkaoui, D. Mammass, The granularity approach of learning objects to support adaptability in adaptive learning systems. Journal of Theoretical and Applied Information Technology. Vol 18. No. $1-2010$.

[29] Amal Battou and al, "Towards an adaptive learning system based on a new learning object granularity approach, international journal of advanced computer science and application, Vol.2 NO. 9, 2011

[30] Maria sette and Lixin Tao, "A semantic approach to intelligent and adaptive learning based on web learning objects, Proceedings of
Student-Faculty Research Day, CSIS, Pace University, May 3rd, 2013.

[31] Sotirios Botsios and Dimitrios Georgiou, "Using standards for adaptive learning retrieval", Departement of Electrical \& computer engineering Democritus Univ. of Thrace, GR 671 00, Xanthi, Greece, 23 jan 2014.

[32] Nassim Matar, Multi adaptive learning objects repository structure towards unified e-learning", International Arab Journal of eTechnology, Vol. 3, No. 3, January 2014.

\section{AUTHORS}

Y. Z. Seghroucheni is with the Faculty of Sciences of Tetouan (e-mail: yassinezaouiseghroucheni@gmail.com).

A. Mohammed, is with the National School of Applied Sciences of Tetouan (e-mail: alachhab@gmail.com).

B. E. El mohajir is the Faculty of Sciences of Tetouan (e-mail : b.elmohajir@ieee.ma)

Submitted 11 April 2014. Published as resubmitted by the authors 14 September 2014. 